\title{
Evaluation of extremely small horizontal emittance
}

\author{
T. Okugi, ${ }^{1}$ T. Hirose, ${ }^{1}$ H. Hayano, ${ }^{2}$ S. Kamada, ${ }^{2}$ K. Kubo, ${ }^{2}$ T. Naito, ${ }^{2}$ K. Oide, ${ }^{2}$ K. Takata, ${ }^{2}$ Seishi Takeda, ${ }^{2}$ \\ N. Terunuma, ${ }^{2}$ N. Toge, ${ }^{2}$ J. Urakawa, ${ }^{2}$ S. Kashiwagi, ${ }^{3}$ M. Takano, ${ }^{4}$ D. McCormick, ${ }^{5}$ M. Minty, ${ }^{5}$ M. Ross, ${ }^{5}$ \\ M. Woodley, ${ }^{5}$ F. Zimmermann, ${ }^{5}$ and J. Corlett ${ }^{6}$ \\ ${ }^{1}$ Faculty of Science, Tokyo Metropolitan University, 1-1 Minami-Osawa, Hachioji, Tokyo 192-0364, Japan \\ ${ }^{2}$ High Energy Accelerator Research Organization, 1-1 Oho, Tsukuba, Ibaraki 305-0801, Japan \\ ${ }^{3}$ The Graduate University for Advanced Studies, 1-1 Oho, Tsukuba, Ibaraki 305-0801, Japan \\ ${ }^{4}$ Faculty of Science, Toho University, 2-2-1 Miyama, Funabashi, Chiba 274-0072, Japan \\ ${ }^{5}$ Stanford Linear Accelerator Center, Stanford University, Stanford, California 94309 \\ ${ }^{6}$ Lawrence Berkeley National Laboratory, Berkeley, California 94720
}

(Received 22 October 1998; published 25 February 1999)

The KEK Accelerator Test Facility (KEK-ATF) was constructed to develop technologies for producing a low-emittance beam which will be required by future linear colliders. The KEK-ATF consists of an injector linac, a damping ring, and a beam extraction line. The basic optical structure of the damping ring is a FOBO lattice, which reduces the horizontal dispersion at the center of the bending magnets and, as a consequence, can produce an extremely small emittance beam. To verify the performance of such a unique, low-emittance lattice, it is crucial to measure the horizontal emittance. The horizontal emittance was measured using wire scanners in the beam extraction line. Since the horizontal beam position was not stable, we established a method to correct the measured beam size for position fluctuation ("jitter") and we succeeded in the observation of the so far smallest horizontal emittance in any accelerator. The measured horizontal emittance was $1.37 \pm 0.03 \mathrm{~nm}$ at a beam energy of $1.285 \mathrm{GeV}$ and a bunch population of $(3-5) \times 10^{9}$, in agreement with the design value of $1.27-$ $1.34 \mathrm{~nm}$ at the beam energy and the bunch population. [S1098-4402(99)00026-9]

PACS numbers: 29.27.Eg, 29.27.Fh, 41.75.Ht

\section{INTRODUCTION}

Third generation synchrotron light sources with small emittances have been constructed for use in physics applications involving synchrotron orbit radiation. The basic optical structure of such accelerators is either a doublebend achromat lattice, for example, at ELETTRA, ESRF, APS, and SPring-8, or a triple-bend achromat lattice, in use at ALS and BESSY II. The natural emittance of the ESRF is $3.0 \mathrm{~nm}$ (for a low-emittance operation ), that of the ALS is $3.4 \mathrm{~nm}$, and those of the others are slightly larger (see Table I). The emittance is defined as

$$
\varepsilon_{x}=\sqrt{\left\langle x^{2}\right\rangle\left\langle x^{\prime 2}\right\rangle-\left\langle x x^{\prime}\right\rangle^{2}},
$$

where the bracket indicates an average over all of the particles and the natural emittance is the steady state emittance in the ring. However, future linear colliders [1,2] will require even smaller emittances than these synchrotron light sources in order to achieve the design luminosity. The damping ring of the KEK Accelerator Test Facility (KEKATF) has been constructed to develop the technologies for producing such beams of unprecedentedly low emittance [3]. The beam operation of the KEK-ATF damping ring began in January, 1997. The KEK-ATF damping ring employs a FOBO lattice with defocusing combined function bending magnets, in order to reduce the horizontal dispersion at the radiation source. The optics of a normal arc cell is shown in Fig. 1 [4]. The principal parameters of the KEK-ATF damping ring are listed in Table II. The design natural emittance of the damping ring is $1.12 \mathrm{~nm}$, which is significantly smaller than that of the third generation synchrotron light sources listed in Table I. It is crucial to reliably measure the horizontal emittance in order to verify the performance of such a unique, low-emittance lattice.

It has been reported that a small beam emittance was measured at several light sources using synchrotron radiation. For example, at the ESRF horizontal and vertical emittances of 3.5 and $0.04 \mathrm{~nm}$, respectively, were measured with an x-ray pinhole camera [11]. It is difficult to accurately measure the horizontal emittance by utilizing the synchrotron radiation in the damping ring because energy spread and dispersion can contribute significantly to the horizontal spot size at the location of the synchrotron light monitor. The KEK-ATF, however, has

TABLE I. Natural emittances for the third generation synchrotron light sources of the world. Lattice type DBA means double-bend achromat lattice and TBA means triple-bend achromat lattice.

\begin{tabular}{lccc}
\hline \hline $\begin{array}{c}\text { Accelerator } \\
\text { name }\end{array}$ & $\begin{array}{c}\text { Lattice } \\
\text { type }\end{array}$ & $\begin{array}{c}\text { Beam } \\
\text { energy }\end{array}$ & $\begin{array}{c}\text { Design } \\
\varepsilon_{x}\end{array}$ \\
\hline ELETTRA (Italy) & DBA & $1.5-2.0 \mathrm{GeV}$ & $7.0 \mathrm{~nm} \mathrm{[5]}$ \\
ESRF (France) & DBA & $6.0 \mathrm{GeV}$ & $3.0-7.0 \mathrm{~nm} \mathrm{[6]}$ \\
APS (USA) & DBA & $7.0 \mathrm{GeV}$ & $8.2 \mathrm{~nm} \mathrm{[7,8]}$ \\
SPring-8 (Japan) & DBA & $8.0 \mathrm{GeV}$ & $5.5 \mathrm{~nm} \mathrm{[9]}$ \\
BESSY II (Germany) & TBA & $1.7 \mathrm{GeV}$ & $5.0 \mathrm{~nm} \mathrm{[10]}$ \\
ALS (USA) & TBA & $1.3-1.9 \mathrm{GeV}$ & $3.4 \mathrm{~nm} \mathrm{[7]}$ \\
\hline \hline
\end{tabular}




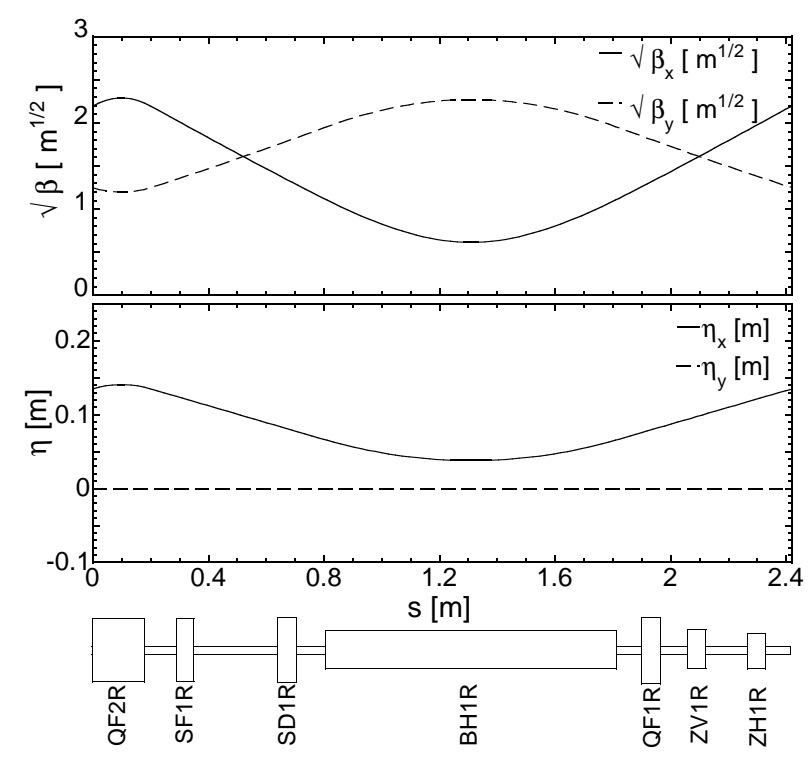

FIG. 1. Normal FOBO cell of the KEK-ATF damping ring. QF2R and BH1R are the main focusing quadrupole magnet and the combined function bending magnet. QF1R is the quadrupole magnet to adjust the defocusing component of the BH1R. The initials " $S$ " and " $Z$ " denote the sextupole magnets and the steering magnets, respectively.

a beam extraction line, whose purpose is to examine the properties of the beam. The beam diagnosis in the extraction line started in November, 1997. In the following sections, we will discuss the emittance measurement apparatus and technique and cures for several types of systematic measurement errors. Finally, we will evaluate and discuss the measurement results.

\section{KEK-ATF EXTRACTION LINE}

Figure 2 depicts the layout of the KEK-ATF extraction line, which consists of five bending magnets and 16 quadrupole magnets. The double kicker system, in which

TABLE II. Design parameters of the ATF damping ring.

\begin{tabular}{lcc}
\hline \hline \multicolumn{1}{c}{ Parameter } & Symbol & Value \\
\hline Beam energy & $E_{\text {beam }}$ & $1.285 \mathrm{GeV}$ \\
Ring circumference & $L_{C}$ & $138.6 \mathrm{~m}$ \\
rf frequency & $f_{\mathrm{RF}}$ & $713.996 \mathrm{MHz}$ \\
rf voltage & $V_{\mathrm{RF}}$ & $283.6 \mathrm{kV}$ \\
Harmonic number & $h$ & 330 \\
Synchrotron frequency & $f_{s}$ & $10.65 \mathrm{kHz}$ \\
Momentum compaction & $\alpha_{\mathrm{M}}$ & $2.141 \times 10^{-3}$ \\
rf momentum acceptance & $\left(\sigma_{p} / p\right)_{\mathrm{RF}}$ & 0.01246 \\
Natural emittance & $\varepsilon_{x}$ & $1.122 \mathrm{~nm}$ \\
Natural bunch length & $\sigma_{z}$ & $5.211 \mathrm{~mm}$ \\
Natural momentum spread & $\sigma_{E} / E$ & $5.434 \times 10^{-4}$ \\
Average beta functions & $\left\langle\beta_{x}\right\rangle$ & $3.869 \mathrm{~m}$ \\
& $\left\langle\beta_{y}\right\rangle$ & $4.459 \mathrm{~m}$ \\
\hline \hline
\end{tabular}

the kicker magnets are separated by a horizontal phase advance of $180^{\circ}$, was chosen to study the suppression of beam position jitter by such a kicker system [12]. At the end of the extraction line there is a beam diagnostic section. The system was designed such that the dispersion is zero in this diagnostic section.

An integrating current transformer (ICT) and 14 single path strip-line-type beam position monitors (BPMs) for beam current and beam position measurement are located in the extraction line. Clipping circuits $[13,14]$ are used to read out the signals from all the BPMs, resulting in a position resolution of approximately $20-30 \mu \mathrm{m}$. The BPM resolution was estimated from the noise of the readout electronics. The resolution of $20-30 \mu \mathrm{m}$ was verified in the beam diagnostic section by repeatedly measuring the jitter-free vertical orbit.

In order to measure the beam size and to evaluate the beam emittance, we installed four wire scanners. Each wire mount is placed at a $45^{\circ}$ tilt angle to the horizontal plane, as shown in Fig. 3. Both the horizontal and vertical beam sizes were measured by sliding the wire mount along its axis and detecting the $\gamma$ rays that are scattered on the wires parallel to the vertical and horizontal plane, respectively. The wire position is read out by measuring the wire mount position using a digital position gauge with a resolution of $5 \mu \mathrm{m}$. This corresponds to a horizontal beam position of approximately $3.5 \mu \mathrm{m}$. Assuming the beam profile is a Gaussian distribution of rms size $\sigma_{\text {real }}$, the measured beam size $\sigma_{\text {meas }}$ is given by

$$
\sigma_{\text {meas }}=\sqrt{\sigma_{\text {real }}^{2}+\left(\frac{d_{\text {wire }}}{4}\right)^{2}},
$$

where $d_{\text {wire }} / 4$ is the second-order moment of a roundshaped wire with a diameter $d_{\text {wire }}$. Since each wire scanner consists of a $50 \mu \mathrm{m}$ diameter tungsten filament, the contribution of the wire thickness to the measured rms beam size is $12.5 \mu \mathrm{m}$. The minimum beam size for all emittance measurements was approximately $30 \mu \mathrm{m}, 3$ times larger than the wire contribution to the beam size measurement. The $\gamma$ rays scattered from a wire scanner are detected by an air Čerenkov detector followed by a photomultiplier (PMT), developed at the Stanford Linear Accelerator Center (SLAC). The air Čerenkov detector is fairly immune to the effects of the background photons, since the detector's threshold energy is set to be $21-22 \mathrm{MeV}$, while the critical energy of the synchrotron radiation produced by the bending magnets in the extraction line is very low at approximately $1 \mathrm{keV}$.

All of these beam monitors are read out on a single beam passage with analog-to-digital converter (ADC) CAMAC modules and the readout signals of each monitor are simultaneously recorded in the VSYSTEM [15] database, thus enabling us to examine any correlations between these monitors. 


\section{ATF Beam Extraction Line}

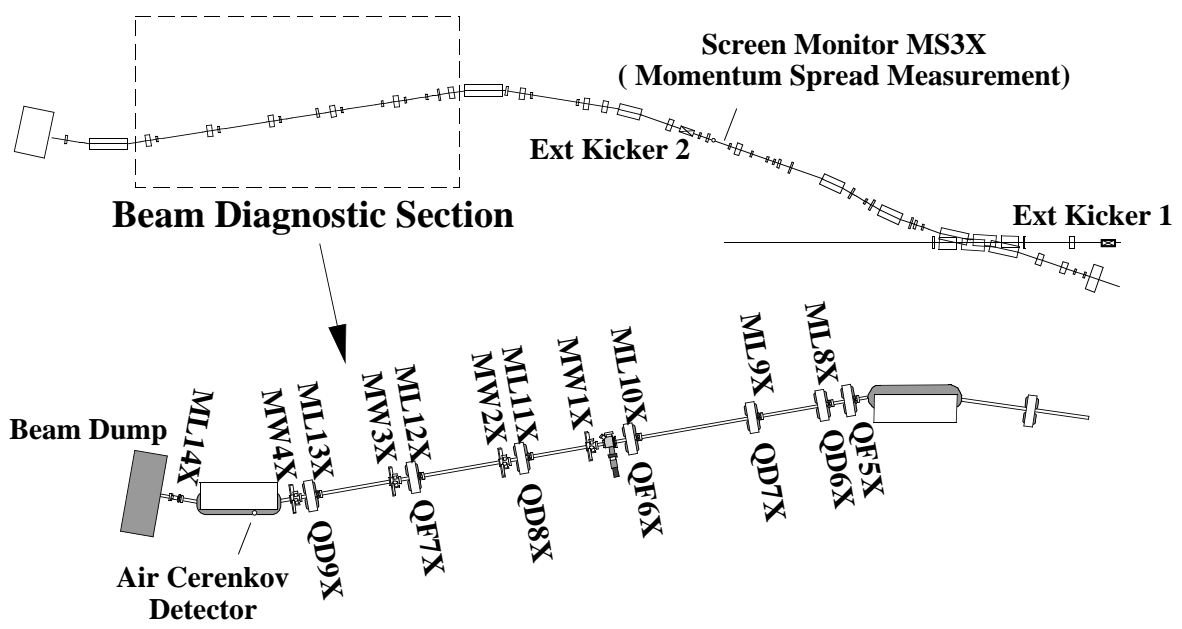

FIG. 2. Layout of the KEK-ATF extraction line. The initial "Q" shows the quadrupole magnets, "MW" the wire scanners, and "ML" the strip-line-type BPMs.

\section{HORIZONTAL DISPERSION CORRECTION}

The general expression for the horizontal beam size $\sigma_{x}$ is

$$
\sigma_{x}=\sqrt{\varepsilon_{x} \beta_{x}+\left(\eta_{x} \frac{\sigma_{p}}{p}\right)^{2}},
$$

with $\varepsilon_{x}$ the horizontal emittance, $\beta_{x}$ the horizontal beta function, $\eta_{x}$ the horizontal dispersion function, and $\sigma_{p} / p$ the relative momentum spread. All wire scanners are located in the dispersion-free diagnostic section mentioned above. However, because the KEKATF damping ring is a test accelerator, magnet strengths, especially those of steering magnets, are often changed for various beam studies. This results in changes of the horizontal dispersion in the damping ring as well as in the extraction line. Since the residual horizontal dispersion in the beam diagnostic section contributes to the measured beam size and the longitudinal beam oscillation gives rise to additional horizontal position jitter proportional to the

\section{Wire scanner chamber}
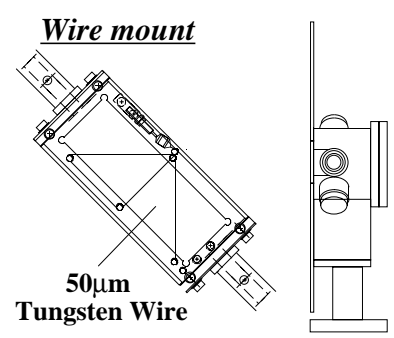

Side View

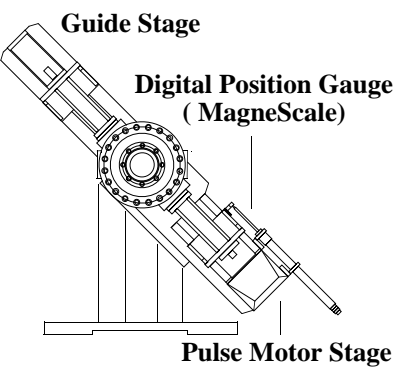

Front View
FIG. 3. Schematic figure of wire scanner used in KEK-ATF. dispersion, we must correct the horizontal dispersion prior to any beam diagnosis in the extraction line.

The horizontal dispersion function in the extraction line is evaluated by measuring the orbit differences for different damping ring rf frequencies. When a beam is injected into the damping ring, the damping ring rf must be synchronized with the linac rf. However, between two successive injections the damping ring of frequency can be changed, in order to measure dispersion function. The frequency shift changes the energy of the extracted beam by

$$
\frac{\Delta p}{p}=-\frac{1}{\alpha_{\mathrm{M}}} \frac{\Delta f_{\mathrm{RF}}}{f_{\mathrm{RF}}}
$$

with $\alpha_{\mathrm{M}}$ the momentum compaction factor and $f_{\mathrm{RF}}$ the rf frequency of the damping ring cavities. The horizontal orbit difference is proportional to the magnitude of the energy shift as

$$
\Delta x=\eta_{x}\left(\frac{\Delta p}{p}\right)=-\frac{\eta_{x}}{\alpha_{\mathrm{M}}} \frac{\Delta f_{\mathrm{RF}}}{f_{\mathrm{RF}}}
$$

ignoring higher-order effects such as second-order dispersion. Therefore, we can evaluate the horizontal dispersion as

$$
\frac{\eta_{x}}{\alpha_{\mathrm{M}}}=-f_{\mathrm{RF}} \frac{\Delta x}{\Delta f_{\mathrm{RF}}} .
$$

Equation (6) demonstrates that the resolution of the dispersion measurement is determined by the position resolution, due to orbit jitter and BPM readout resolution, and by the magnitude of the rf frequency change. A large rf frequency change improves the resolution of the dispersion measurement even when the BPM readout resolution is poor or a large position jitter exists. The magnitude of the $r f$ frequency change is limited by the vacuum chamber diameter of $24 \mathrm{~mm}$ and a large horizontal dispersion with 
a maximum value of approximately $2.5 \mathrm{~m}$. To allow the beam through a vacuum chamber without beam loss in the large dispersion region, the rf frequency change must be smaller than $\pm 4.7 \mathrm{kHz}$. In addition, a linearity range of $3 \mathrm{~mm}$ for the BPM readout electronics [13,14] further restricts the useful range of $\mathrm{rf}$ frequencies to approximately $\pm 2 \mathrm{kHz}$. Abandoning the dispersion measurement in the large dispersion region would improve the resolution of the dispersion measurement at other BPM locations by a factor of 2 or more. However, the dispersion measurement resolution for $\Delta f_{\mathrm{RF}}= \pm 2 \mathrm{kHz}$ is $11 \mathrm{~mm}$, even if we assume the beam position jitter of $200 \mu \mathrm{m}$ and 30 times data taking, and it corresponds to a beam size of $6.8 \mu \mathrm{m}$. This is adequate for the dispersion correction because the typical horizontal beam size in the beam diagnostic section is $30-100 \mu \mathrm{m}$. Therefore, the maximum rf frequency change for the horizontal dispersion measurement was limited to $\pm 2 \mathrm{kHz}$.

The horizontal dispersion measurement and its correction were carried out as follows. First, the beam orbits for five rf frequency offsets, $+2 \mathrm{kHz},+1 \mathrm{kHz}, 0 \mathrm{kHz}$, $-1 \mathrm{kHz}$, and $-2 \mathrm{kHz}$, were measured randomly by the BPMs pulse by pulse to reduce the systematic error be-

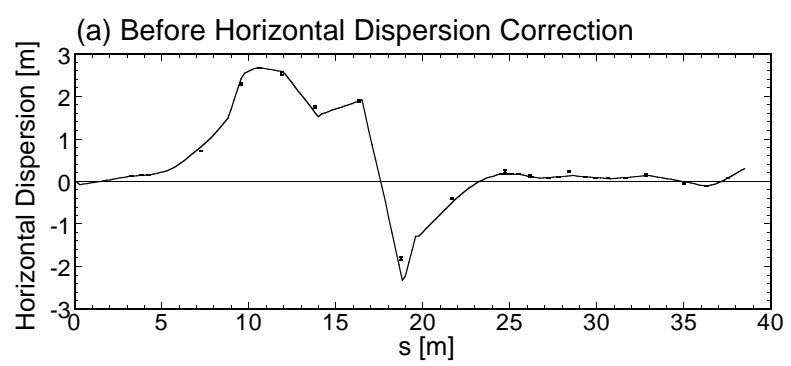

(b) After Horizontal Dispersion Correction
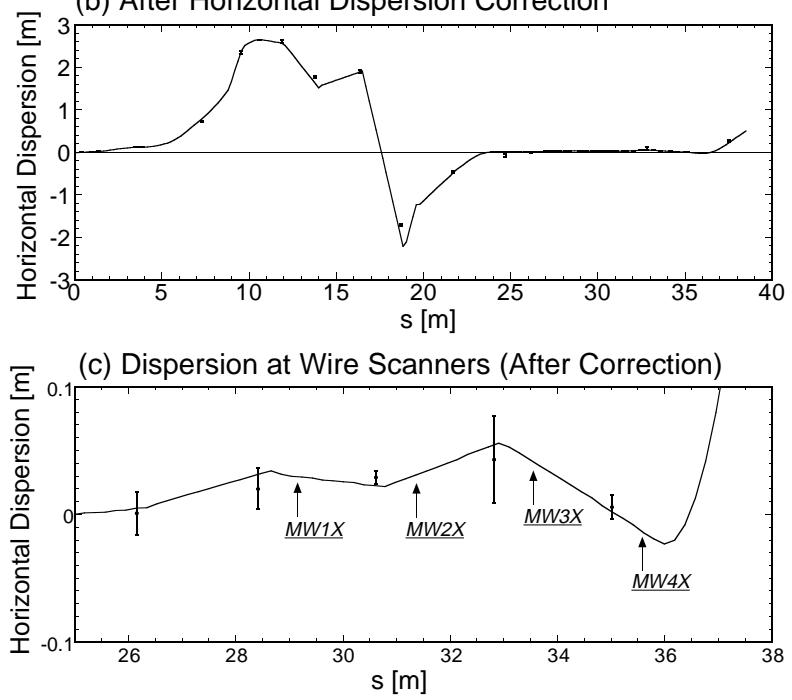

FIG. 4. Horizontal dispersion (a) before the dispersion correction, (b) after the dispersion correction, and (c) at the wire scanner location (after the dispersion correction). The points show the measured dispersion for each BPM position and the lines show the fitted results. tween the measurement with each offset such as orbit drift. The position was measured approximately 30 times for each rf frequency offset. We then evaluated the slopes $\Delta x_{\text {ramp }} / \Delta f_{\text {RF }}$ by linear fitting using a MINUIT [16] software package and obtained the horizontal dispersion for each BPM position applying Eq. (6). The horizontal dispersion at the wire scanner locations was evaluated by fitting for the two parameters $\eta_{x}, \eta_{x}^{\prime}$ at the entrance of the extraction line. For the fit, we utilized a linear optics model of the extraction line, which includes the effect of finite magnet thickness. Finally, we calculated the magnet settings required to suppress the horizontal dispersion in the beam diagnostic section and changed the strengths of some quadrupole magnets accordingly. An example of the measured horizontal dispersion before and after correction is shown in Fig. 4. After the correction, the value of the horizontal dispersion is reduced within $\pm 5 \mathrm{~cm}$.

\section{POSITION JITTER CORRECTION}

Since one of the extraction kickers suffered from discharges, the horizontal beam position of the extracted beam was not stable. Because the magnitude of the orbit fluctuation of 50-200 $\mu \mathrm{m}$ was comparable to the typical horizontal beam size of $30-100 \mu \mathrm{m}$, we applied a position jitter correction method to infer the real beam size. The position correction was not carried out using all BPMs on the extraction line since the resolution obtained with two BPMs was adequate and allowed for a fast calculation in the on-line control system. In a dispersion-free section, the horizontal beam offset at an arbitrary position $x_{3}$ is calculated from the horizontal offsets at two other positions $x_{1}, x_{2}$ as follows:

$$
x_{3}=\frac{R_{12}\left(s_{3}, s_{1}\right)}{R_{12}\left(s_{2}, s_{1}\right)} x_{2}-\frac{R_{12}\left(s_{3}, s_{2}\right)}{R_{12}\left(s_{2}, s_{1}\right)} x_{1} .
$$

Here, $R_{12}\left(s_{j}, s_{i}\right)$ is a transfer matrix component from $s_{i}$ to $s_{j}$. The transfer matrix elements were obtained using the measured magnetic field strengths. As the horizontal beam offsets at more than two positions are measured by BPMs, we can estimate the beam offset at any position. The expected position resolution $\sigma_{x_{3}}$ depends upon the BPM readout resolution $\sigma_{x, \text { BPM }}$ as

$$
\begin{aligned}
\sigma_{x_{3}} & =\sqrt{\left(\frac{R_{12}\left(s_{3}, s_{1}\right)}{R_{12}\left(s_{2}, s_{1}\right)}\right)^{2}+\left(\frac{R_{12}\left(s_{3}, s_{2}\right)}{R_{12}\left(s_{2}, s_{1}\right)}\right)^{2}} \sigma_{x, \mathrm{BPM}} \\
& =f\left(s_{1}, s_{2}, s_{3}\right) \sigma_{x, \mathrm{BPM}} .
\end{aligned}
$$

While any set of two BPM readouts can be used for $x_{1}, x_{2}$ in Eq. (7), the set of BPMs should be chosen to minimize the resolution factor $f\left(s_{1}, s_{2}, s_{3}\right)$ for each relevant location $s_{3}$.

In preparation for the position correction of the beam size measurement using Eq. (7), the quality of the position estimate was tested by applying the estimate to the 
TABLE III. The list of resolution factors for each monitor located at the beam diagnostic section. BPM1, BPM2 are the set of BPMs for minimum resolution factors. The monitor type BPM stands for a beam position monitor and WS means wire scanner.

\begin{tabular}{ccclc}
\hline \hline Monitor name & Type & BPM1 & BPM2 & $f\left(s_{1}, s_{2}, s_{3}\right)$ \\
\hline ML10X & BPM & ML14X & ML8X & 0.634 \\
ML11X & BPM & ML12X & ML14X & 0.293 \\
ML12X & BPM & ML14X & ML8X & 1.249 \\
ML13X & BPM & ML8X & ML12X & 0.196 \\
MW1X & WS & ML11X & ML10X & 0.758 \\
MW2X & WS & ML12X & ML10X & 0.432 \\
MW3X & WS & ML12X & ML9X & 0.868 \\
MW4X & WS & ML10X & ML11X & 0.370 \\
\hline \hline
\end{tabular}

BPM location, where it could be compared with a direct BPM measurement. The set of BPMs used for the position estimation and the corresponding resolution factor $f\left(s_{1}, s_{2}, s_{3}\right)$ for each BPM is listed in Table III. The BPMs used for the position correction were chosen so as to obtain the best resolution. Using Eq. (7), we define a quantity $X\left(s_{3}\right)$ as

$$
X\left(s_{3}\right)=x_{3}-\left(\frac{R_{12}\left(s_{3}, s_{1}\right)}{R_{12}\left(s_{2}, s_{1}\right)} x_{2}-\frac{R_{12}\left(s_{3}, s_{2}\right)}{R_{12}\left(s_{2}, s_{1}\right)} x_{1}\right) .
$$

$X\left(s_{3}\right)$ would ideally be zero for all BPM locations, but an off-center beam orbit at a quadrupole magnet between $s_{1}$ and $s_{3}$ might cause a finite value for $X\left(s_{3}\right)$, due to the kick of the quadrupole magnet. The fluctuation of $X\left(s_{3}\right)$, however, should be given by

$$
\sigma_{X}\left(s_{3}\right)=\sqrt{1+f^{2}\left(s_{1}, s_{2}, s_{3}\right)} \sigma_{x, \mathrm{BPM}}
$$

even if a huge position jitter exists. Figure 5 shows a typical result of the position estimation at a BPM location. The individual BPM readout fluctuation was 50-200 $\mu \mathrm{m}$, while the measured $\sigma_{X}\left(s_{3}\right)$ 's of 30-40 $\mu \mathrm{m}$ for all BPMs were in good agreement with the expected 20-30 $\mu \mathrm{m}$ fluctuation of the BPM readout. Note that some deviations from Eq. (10) are explained by nonzero dispersion at two of the BPMs used for position correction

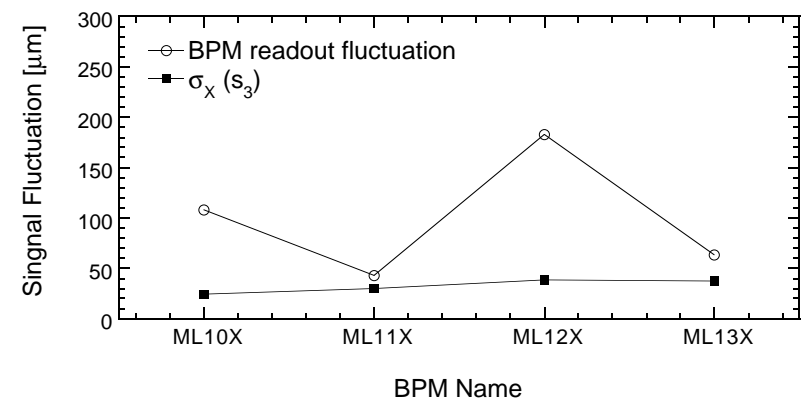

FIG. 5. Result of a performance test of position prediction for BPMs located at a beam diagnostic section. The circles show BPM readout fluctuations and the squares show $\sigma_{X}\left(s_{3}\right)$ defined in Eq. (10). at the BPM locations. For correcting the beam position at the wire scanners, only dispersion-free BPMs are being employed.

Next, we applied the position estimation method to the beam size measurement with wire scanners. The set of BPMs used for determining the beam position and the corresponding resolution factor $f\left(s_{1}, s_{2}, s_{3}\right)$ for each wire scanner is also listed in Table III. As mentioned above, the horizontal beam position at the wire scanners fluctuated pulse by pulse. We can derive the beam position with respect to the wire $x_{\mathrm{WS}}$ by using the position estimation of Eq. (7)

$$
x_{\mathrm{WS}}=\frac{x_{\mathrm{DG}}}{\sqrt{2}}-\left(\frac{R_{12}\left(s_{\mathrm{WS}}, s_{1}\right)}{R_{12}\left(s_{2}, s_{1}\right)} x_{2}-\frac{R_{12}\left(s_{\mathrm{WS}}, s_{2}\right)}{R_{12}\left(s_{2}, s_{1}\right)} x_{1}\right),
$$

with $s_{\mathrm{WS}}$ the wire scanner position and $x_{\mathrm{DG}}$ the digital position gauge readout on the wire scanner, which was divided by $\sqrt{2}$ to account for the $45^{\circ}$ tilt (see Fig. 3). A typical ADC readout of air Čerenkov detector PMT signals is shown in Fig. 6(a) as a function of the uncorrected digital gauge readout position $x_{\mathrm{DG}}$ indicating that PMT signals fluctuate over a wide range. Figure 6(b) shows PMT signals as a function of the calculated beam position with respect to the wire $x_{\mathrm{WS}}$, demonstrating that our correction algorithm worked well. Figure 6 shows the results demonstrated with the wire scanner MW1X.

The rms size obtained from the Gaussian fit to the PMT signals as a function of the beam position with respect to the wire $x_{\text {WS }}$ was taken to be the beam size at the wire scanner location. Since other contributions of systematic errors were negligibly small, the error of the Gaussian fit was used as an estimate of the measurement error, which typically was $1-5 \mu \mathrm{m}$.

\section{HORIZONTAL EMITTANCE EVALUATION}

The measured horizontal beam size is determined not only by the horizontal emittance and horizontal beta function $\sigma_{x \beta}$, but it also contains contributions from the finite wire diameter, as shown in Eq. (2), and from the residual horizontal dispersion at the wire location. The component of the horizontal beam size arising from emittance and beta function is obtained as

$$
\sigma_{x \beta} \simeq \sqrt{\sigma_{x}^{2}-\left(\frac{d_{\text {wire }}}{4}\right)^{2}-\left(\eta_{x} \frac{\sigma_{p}}{p}\right)^{2}},
$$

with $d_{\text {wire }}$ the wire diameter and $\sigma_{x}$ the measured horizontal beam size. The corresponding error is expressed as

$$
\begin{aligned}
\Delta \sigma_{x \beta}^{2} \simeq & {\left[\frac{\sigma_{x}}{\sigma_{x \beta}} \Delta \sigma_{x}\right]^{2}+\left[\frac{\eta_{x}\left(\sigma_{p} / p\right)^{2}}{\sigma_{x \beta}} \Delta \eta_{x}\right]^{2} } \\
& +\left[\frac{\eta_{x}^{2}\left(\sigma_{p} / p\right)}{\sigma_{x \beta}} \frac{\Delta \sigma_{p}}{p}\right]^{2}
\end{aligned}
$$


(a) Not Corrected Profile

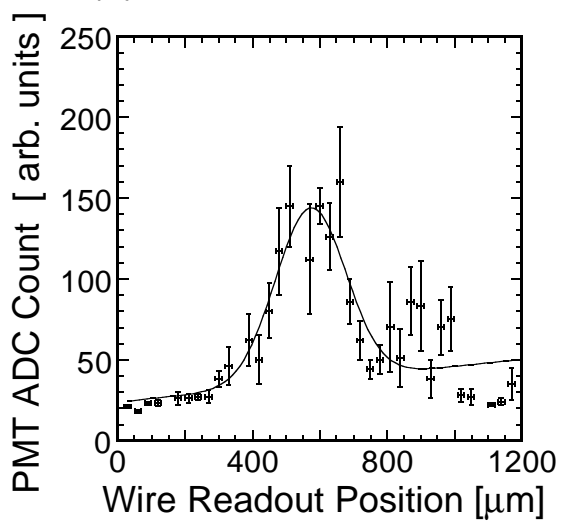

(b) Corrected Profile

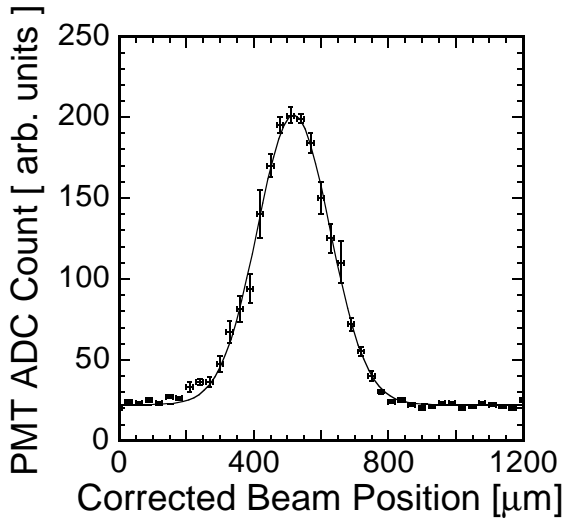

FIG. 6. Result of a position jitter correction. (a) PMT signals as a function of wire readout position and (b) PMT signals as a function of the beam position with respect to the wire. The data were taken 10 pulses for one wire position.

with $\Delta \sigma_{x}$ the error of beam size measurement, $\Delta \eta_{x}$ the error of horizontal dispersion function, and $\Delta\left(\sigma_{p} / p\right)$ the error of momentum spread. Since the deviation from the estimated wire contribution as $d_{\text {wire }} / 4$ is negligibly small for the measured beam size, the contribution of the wire diameter correction can be ignored. The momentum spread of the extracted beam was measured with a screen monitor MS3X located at a position with approximately $1.7 \mathrm{~m}$ dispersion. The result of the momentum spread measurement is shown as a function of beam intensity in Fig. 7. The momentum spread at zero current, i.e., $\sigma_{p} / p=5.5 \pm 0.2 \times 10^{-4}$, agreed well with a design value of $5.43 \times 10^{-4}$. However, the momentum spread increased with beam intensity. During the emittance measurement, the beam intensity changed pulse by pulse, with a typical bunch population of $(3-5) \times 10^{9}$. Therefore, we assumed that the momentum spread at the time of the horizontal beam size measurement was $6.0 \pm 0.3 \times 10^{-4}$.

Two different methods are used for evaluating the horizontal emittance: one is the waist-scan method, and the other is the four-wire method. In the waist-scan method, the beam emittance is determined by measuring the beam size with a single wire scanner while changing the strength of a quadrupole magnet located upstream of the monitor. We performed the waist scans on the three wire scanners MW1X, MW2X, and MW3X. The waist-scan method was not carried out for MW4X because the horizontal beam size at MW4X was insensitive to a strength change in the upstream quadrupole magnet.

The spot sizes measured at each wire scanner during the waist scans had both betatron and dispersive contributions, since a small residual dispersion still remained at the wire scanner locations even after dispersion correction. (In Sec. III we demonstrated that the residual dispersion was less than $\pm 5 \mathrm{~cm}$, which corresponds to $30 \mu \mathrm{m}$ of beam size.) In order to remove the dispersive component from the measured spot sizes at each wire scanner, we measured the residual horizontal dispersion at one typical quadrupole strength. After that, we calculated the dispersion at the other quadrupole strength from the measured dispersion at the beam position monitors located upstream of the quadrupole magnet with the linear optics model. We then subtracted the dispersive component from the measured spot sizes using Eq. (12) and the estimated horizontal dispersion (see Table IV). In all cases, the dispersive part of the spot size was less than one-fifth of the betatron size for wire scanner MW1X, and less than one-fourth for wire scanners MW2X and MW3X. By subtracting the contribution of the residual dispersion according to Eq. (12), we determined the beam size component due to beta function and emittance. Figures 8(a)-8(c) show the square of this betatron beam size for MW1X, MW2X, and MW3X as a function of quadrupole strength. The horizontal emittance values inferred from the parabolic fits are summarized in Table V.

A second method to evaluate the beam emittance is the four-wire method. By measuring the beam sizes with four independent wire scanners, and fitting the result to an optics model, the Twiss parameters $\alpha_{x}, \beta_{x}$ and the horizontal emittance can be obtained. A measurement result for the horizontal emittance is shown in Fig. 8(d). The ellipse represents the beam distribution in phase space $\left(x, x^{\prime}\right)$ at the entrance of the extraction line. Four lines show the measured beam size boundaries converted to the same phase space. The corresponding horizontal emittance value is also listed in Table V. For all horizontal emittance measurements, both the waist-scan and four-wire methods agreed within 2 standard deviations. An average horizontal emittance of $1.37 \pm 0.03 \mathrm{~nm}$ was obtained.

The fitted Twiss parameters were used to verify each horizontal emittance evaluation. A mismatch factor $B_{\text {mag, } x}[17,18]$ is defined as

$$
B_{\mathrm{mag}, x}=\frac{1}{2}\left[\frac{\beta_{x 2}}{\beta_{x 1}}+\frac{\beta_{x 1}}{\beta_{x 2}}+\beta_{x 1} \beta_{x 2}\left(\frac{\alpha_{x 1}}{\beta_{x 1}}-\frac{\alpha_{x 2}}{\beta_{x 2}}\right)^{2}\right]
$$



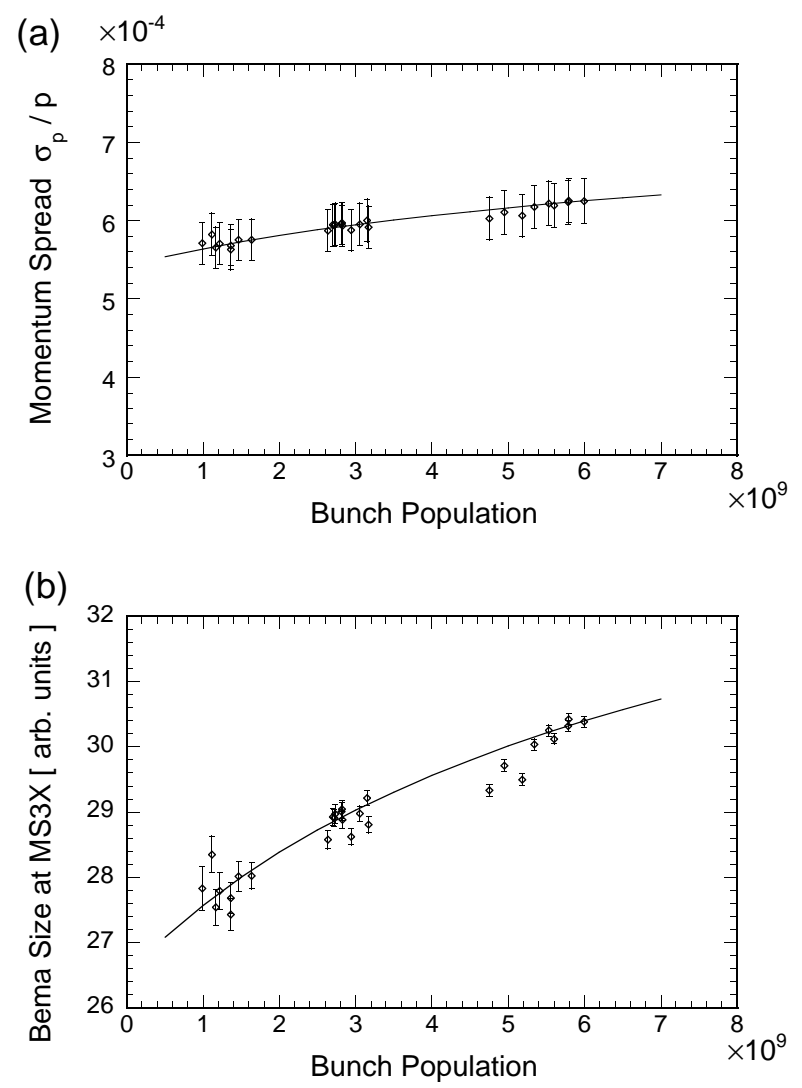

(C)

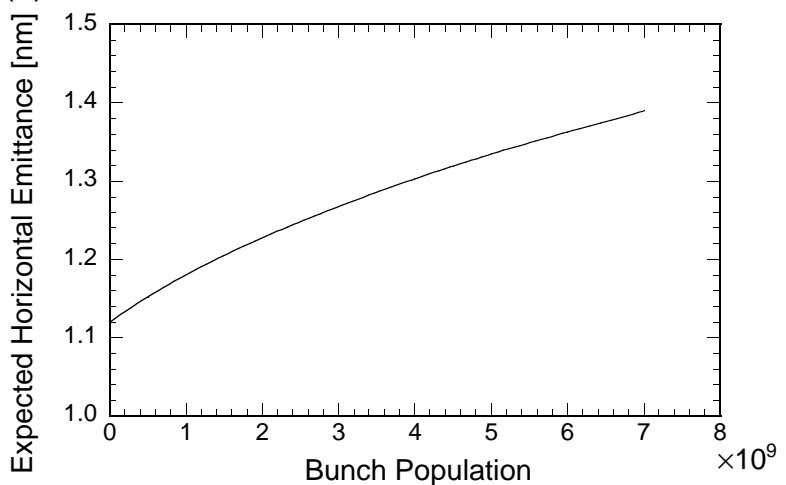

FIG. 7. Results of momentum spread measurement by the screen monitor MS3X. (a) The measured momentum spread, which is evaluated to be $(5.5 \pm 0.2) \times 10^{-4}$ at zero current and $6.0 \pm 0.3 \times 10^{-4}$ at the bunch population of $(3-5) \times 10^{9}$. A scaling error from the charge-coupled device pixel to the beam size of $35.4 \pm 1.6 \mu \mathrm{m} /$ pixel contributes to the large momentum spread error. (b) Vertical axis is changed to a measured beam size at the screen monitor. The curve shows the expected momentum spread, which is assumed to be increased by the intrabeam scattering while keeping a constant emittance ratio $\varepsilon_{x} / \varepsilon_{y}=2.31 \%$. (c) Expected horizontal emittance at the emittance ratio of $2.31 \%$.

for two different sets of Twiss parameters $\alpha_{x}, \beta_{x}$. The beam emittance was evaluated by assuming the transfer matrix from the quadrupole magnet to the wire scanner for the waist-scan method and assuming the transfer matrix between the wire scanners for the four-wire method. We checked the optics error and the consistency of evaluated emittances by using $B_{\mathrm{mag}, x}$. In the calculation of $B_{\mathrm{mag}, x}$, each measurement was used as one set of Twiss parameters, and an average Twiss parameter for the other three measurements weighted by their errors was used as the second set. That all $B_{\text {mag, } x}$ were close to unity shows that the different horizontal emittance measurements were consistent with each other. The extracted values of $B_{\text {mag, } x}$ 's are also shown in Table V.

\section{DISCUSSION}

During the emittance measurement, an intensity dependence of the momentum spread was observed. One possible source of the momentum spread enhancement is the microwave instability $[19,20]$. However, the threshold of the microwave instability for $1.3 \mathrm{GeV}$ beam energy was calculated to be a bunch population of $1.3 \times 10^{10}$ [21]. Since this threshold is 3 times higher than the bunch population during the experiment, it is not a probable reason for the energy spread enhancement.

A more likely reason would be the multiple Touschek effect (intrabeam scattering), which was investigated by Bruck and Le Duff [22,23], in order to explain differences between the measured beam lifetime and the lifetime expected from the single Touschek effect, and generalized by Piwinski [24]. They showed that small momentum transfer from a transverse to a longitudinal direction does not contribute directly to particle losses but acts as an additional excitation source on the momentum spread. As a consequence, they showed it produced a new steady state of the momentum spread. Since the bunch volume in the KEK-ATF damping ring is extremely small, it is expected that an effect of the intrabeam scattering has a strong influence on the momentum spread enhancement [25]. Therefore, we regard it as the main source of the observed momentum spread enhancement. In addition to affecting the momentum spread, the longitudinal momentum impulse at a dispersive position affects the betatron oscillation and transverse equilibrium emittances are also expected to increase with the beam intensity through this intrabeam scattering process. The effect in the KEKATF damping ring was evaluated by the SAD computer code [26] self-consistently taking into account both the momentum spread and the emittances. The calculated momentum spread enhancement was compared to the measured one shown in Fig. 7(b), and the measured intensity dependence on the momentum spread corresponds to the bunch volume with $2.31 \pm 0.68 \%$ emittance coupled beam. The curve in Fig. 7(b) shows the momentum spread, which is assumed to be increased by the intrabeam scattering while keeping the constant emittance ratio $\varepsilon_{x} / \varepsilon_{y}=2.31 \%$ through all the beam current. Furthermore, the corresponding horizontal emittance is expected to be $1.27 \pm 0.04 \mathrm{~nm}$ at the bunch population 
TABLE IV. The list of measured beam sizes and dispersions and estimated betatron beam sizes.



(b) MW2X waist scan

\begin{tabular}{crrr} 
QD7X current & Measured beam size $\sigma_{x}$ & Dispersion $\eta_{x}$ & Estimated betatron beam size $\sigma_{x \beta}$ \\
\hline $5.18 \mathrm{~A}$ & $115.91 \pm 1.69 \mu \mathrm{m}$ & $1.43 \pm 2.26 \mathrm{~cm}^{\mathrm{b}}$ & $114.91 \pm 2.07 \mu \mathrm{m}$ \\
$9.18 \mathrm{~A}$ & $88.02 \pm 1.04 \mu \mathrm{m}$ & $1.41 \pm 2.22 \mathrm{~cm}^{\mathrm{b}}$ & $86.71 \pm 1.68 \mu \mathrm{m}$ \\
$13.18 \mathrm{~A}$ & $63.74 \pm 0.48 \mu \mathrm{m}$ & $1.39 \pm 1.82 \mathrm{~cm}^{\mathrm{b}}$ & $61.95 \pm 1.55 \mu \mathrm{m}$ \\
$17.18 \mathrm{~A}$ & $38.49 \pm 1.77 \mu \mathrm{m}$ & $1.36 \pm 1.43 \mathrm{~cm}^{\mathrm{b}}$ & $35.47 \pm 2.76 \mu \mathrm{m}$ \\
$21.18 \mathrm{~A}$ & $40.80 \pm 0.23 \mu \mathrm{m}$ & $1.34 \pm 1.04 \mathrm{~cm}^{\mathrm{a}}$ & $38.00 \pm 1.34 \mu \mathrm{m}$ \\
$25.18 \mathrm{~A}$ & $60.18 \pm 0.15 \mu \mathrm{m}$ & $1.31 \pm 0.68 \mathrm{~cm}^{\mathrm{b}}$ & $58.34 \pm 0.57 \mu \mathrm{m}$ \\
$29.18 \mathrm{~A}$ & $86.79 \pm 0.77 \mu \mathrm{m}$ & $1.29 \pm 0.44 \mathrm{~cm}^{\mathrm{b}}$ & $85.54 \pm 0.82 \mu \mathrm{m}$ \\
$33.18 \mathrm{~A}$ & $116.98 \pm 0.38 \mu \mathrm{m}$ & $1.26 \pm 0.53 \mathrm{~cm}^{\mathrm{b}}$ & $116.06 \pm 0.44 \mu \mathrm{m}$ \\
$37.18 \mathrm{~A}$ & $149.31 \pm 1.45 \mu \mathrm{m}$ & $1.24 \pm 0.86 \mathrm{~cm}^{\mathrm{b}}$ & $148.60 \pm 1.48 \mu \mathrm{m}$ \\
\hline
\end{tabular}

(c) MW3X waist scan

\begin{tabular}{crrr} 
QF6X current & Measured beam size $\sigma_{x}$ & Dispersion $\eta_{x}$ & Estimated betatron beam size $\sigma_{x \beta}$ \\
\hline $13.85 \mathrm{~A}$ & $177.00 \pm 1.24 \mu \mathrm{m}$ & $4.50 \pm 2.04 \mathrm{~cm}^{\mathrm{b}}$ & $174.48 \pm 2.28 \mu \mathrm{m}$ \\
$15.85 \mathrm{~A}$ & $151.35 \pm 0.32 \mu \mathrm{m}$ & $4.14 \pm 2.28 \mathrm{~cm}^{\mathrm{b}}$ & $148.77 \pm 2.32 \mu \mathrm{m}$ \\
$17.85 \mathrm{~A}$ & $114.78 \pm 0.13 \mu \mathrm{m}$ & $3.93 \pm 2.57 \mathrm{~cm}^{\mathrm{b}}$ & $111.63 \pm 3.27 \mu \mathrm{m}$ \\
$19.85 \mathrm{~A}$ & $86.08 \pm 1.82 \mu \mathrm{m}$ & $3.43 \pm 2.91 \mathrm{~cm}^{\mathrm{b}}$ & $82.64 \pm 4.75 \mu \mathrm{m}$ \\
$21.85 \mathrm{~A}$ & $88.03 \pm 1.07 \mu \mathrm{m}$ & $3.07 \pm 3.28 \mathrm{~cm}^{\mathrm{a}}$ & $85.16 \pm 4.40 \mu \mathrm{m}$ \\
$23.85 \mathrm{~A}$ & $111.84 \pm 0.73 \mu \mathrm{m}$ & $2.71 \pm 3.66 \mathrm{~cm}^{\mathrm{b}}$ & $109.94 \pm 3.33 \mu \mathrm{m}$ \\
$25.85 \mathrm{~A}$ & $143.65 \pm 0.58 \mu \mathrm{m}$ & $2.35 \pm 4.05 \mathrm{~cm}^{\mathrm{b}}$ & $142.41 \pm 2.48 \mu \mathrm{m}$ \\
$27.85 \mathrm{~A}$ & $189.68 \pm 0.67 \mu \mathrm{m}$ & $2.00 \pm 4.46 \mathrm{~cm}^{\mathrm{b}}$ & $188.89 \pm 1.83 \mu \mathrm{m}$ \\
\hline
\end{tabular}

(d) Four-wire method

\begin{tabular}{ccrr} 
Monitor name & Measured beam size $\sigma_{x}$ & Dispersion $\eta_{x}$ & Estimated betatron beam size $\sigma_{x \beta}$ \\
\hline MW1X & $128.53 \pm 0.93 \mu \mathrm{m}$ & $0.75 \pm 0.52 \mathrm{~cm}^{\mathrm{a}}$ & $127.84 \pm 0.94 \mu \mathrm{m}$ \\
MW2X & $40.80 \pm 0.23 \mu \mathrm{m}$ & $1.34 \pm 1.04 \mathrm{~cm}^{\mathrm{a}}$ & $38.00 \pm 1.34 \mu \mathrm{m}$ \\
MW3X & $88.03 \pm 1.07 \mu \mathrm{m}$ & $3.07 \pm 3.28 \mathrm{~cm}^{\mathrm{a}}$ & $85.16 \pm 4.40 \mu \mathrm{m}$ \\
MW4X & $55.50 \pm 0.48 \mu \mathrm{m}$ & $-3.98 \pm 4.83 \mathrm{~cm}^{\mathrm{a}}$ & $48.52 \pm 13.70 \mu \mathrm{m}$ \\
\hline
\end{tabular}

${ }^{a}$ Measured horizontal dispersion.

${ }^{\mathrm{b}}$ Estimated horizontal dispersion from the dispersion with a linear optics model.

of $3 \times 10^{9}$ and $1.34 \pm 0.05 \mathrm{~nm}$ at $5 \times 10^{9}$ by the multiple Touschek effect [see Fig. 7(c)]. Thus the expected horizontal emittance at the beam intensity was in agreement with that of $1.37 \pm 0.03 \mathrm{~nm}$ inferred from the beam size measurements.

\section{CONCLUSION}

The KEK-ATF has been constructed to develop the technologies for producing the low-emittance beam required by future linear colliders. The design natural emit- tance in the damping ring is extremely small, namely $1.12 \mathrm{~nm}$. The basic optical structure of the damping ring is a FOBO lattice, with a small horizontal dispersion at the center of the bending magnets. It is crucial to accurately determine the horizontal emittance in order to examine the performance of such a unique, low-emittance lattice. From the position corrected beam size measurements in the extraction line, we inferred a horizontal emittance of $1.37 \pm 0.03 \mathrm{~nm}$. This value is slightly larger than the design natural emittance. However, the difference of $15 \%-20 \%$ can be attributed to the multiple Touschek 



FIG. 8. Beam-size measurement result. (a), (b), and (c) are results of waist scans for each monitor, and (d) shows the result of a four-wire measurement.

TABLE V. The results of horizontal emittance measurements. "Average" is the data average weighted by the errors.

\begin{tabular}{|c|c|c|c|}
\hline Method & Monitor & $\begin{array}{c}\text { Emittance } \\
(\mathrm{nm})\end{array}$ & $B_{\text {mag }, x}$ \\
\hline Waist-scan & MW1X & $1.47 \pm 0.06$ & $1.03 \pm 0.07$ \\
\hline Waist-scan & MW2X & $1.27 \pm 0.06$ & $1.00 \pm 0.03$ \\
\hline Waist-scan & MW3X & $1.38 \pm 0.05$ & $1.02 \pm 0.05$ \\
\hline Four-wire & All monitors & $1.29 \pm 0.11$ & $1.06 \pm 0.34$ \\
\hline Average & & $1.37 \pm 0.03$ & $\alpha_{x}: 3.83 ; \beta_{x}: 6.77$ \\
\hline
\end{tabular}

effect. For a bunch population of $3-5 \times 10^{9}$, the horizontal emittance is expected to be $1.27-1.34 \mathrm{~nm}$, in agreement with the measured values. It was verified that the FOBO lattice is a valuable lattice for the generation of extremely small emittance beams.

\section{ACKNOWLEDGMENTS}

The authors express their thanks to Professor H. Sugawara, Professor Y. Kimura, Professor M. Kihara, Professor S. Iwata, Professor G. Loew, Professor E. Paterson, Professor D. Burke, and Professor J. Sheppard for their continuous encouragement and support in this work. The authors would especially like to thank to Dr. M. Akemoto, Dr. S. Araki, Dr. Y. Funahashi, Dr. F. Hinode, Dr. T. Ko- rhonen, Dr. S. Sakanaka, and Dr. N. Sato for their contribution of the hardware development, and Dr. M. Kikuchi, Dr. S. Kuroda, and Dr. T. Raubenheimer for their design works of KEK-ATF. Finally, the authors thank all other KEK-ATF Collaboration members in BINP, CERN, DESY, IHEP, KEK, LBNL, PAL, SLAC, Kyoto University, Science University of Tokyo, Tohoku-Gakuin University, Tohoku University, Tokyo Metropolitan University, and University of Tokyo.

[1] JLC Design Study Group, KEK Report No. 97-1, 1997.

[2] The NLC Design Group, LBNL-PUB-524, SLAC Report No. 474, UCRL-ID-12416, 1996.

[3] ATF Design and Study Report, KEK Internal Report No. 95-4, 1995, edited by F. Hinode, S. Kawabata, H. Matsumoto, K. Oide, K. Takata, Seishi Takeda, and J. Urakawa.

[4] J. Urakawa et al., Int. J. Mod. Phys. A (Proc. Suppl.) 2, 124 (1993).

[5] Carlo J. Bocchetta, in Proceedings of the Fifth European Particle Accelerator Conference, Barcelona, 1996 (Institute of Physics, Bristol, UK, 1996), p. 76.

[6] A. Ropert and L. Farvacque, in Proceedings of the 1997 Particle Accelerator Conference, Vancouver, Canada, 1997 (IEEE, Piscataway, NJ, 1998), p. 754. 
[7] Glenn Decker, in Proceedings of the Fifth European Particle Accelerator Conference, Barcelona, 1996 (Institute of Physics, Bristol, UK, 1996), p. 95.

[8] Glenn Decker, in Proceedings of the 1997 Particle Accelerator Conference, Vancouver, Canada, 1997 (IEEE, Piscataway, NJ, 1998), p. 698.

[9] H. Kamitsubo, Proceedings of the 1997 Particle Accelerator Conference, Vancouver, Canada, 1997 (IEEE, Piscataway, NJ, 1998), p. 6.

[10] BESSY-II Project Team, E. Jaeschke, in Proceedings of the 1997 Particle Accelerator Conference, Vancouver, Canada, 1997 (IEEE, Piscataway, NJ, 1998), p. 713.

[11] P. Elleaume, C. Fortgang, C. Penel, and E. Tarazona, J. Synchrotron Radiat. 2, 209 (1995).

[12] H. Nakayama, in Proceedings of the SLAC/KEK Linear Collider Workshop on Damping Ring, Tsukuba, Japan, 1992, KEK Proceedings 92-6 (KEK, Tsukuba, Japan, 1992), p. 326.

[13] F. Hinode, J. Urakawa, and Y. Yoribayashi, in Proceedings of the 1997 Particle Accelerator Conference, Vancouver, Canada, 1997 (IEEE, Piscataway, NJ, 1998), p. 578.

[14] M. Takano, H. Hayano, J. Urakawa, and T. Okugi, Proceedings of the Sixth European Particle Accelerator Conference, Stockholm, 1998 (Institute of Physics, Bristol, UK, 1998), p. 1607.

[15] VSYSTEM is a commercial product of Vista-Control Systems, Inc., Los Alamos, New Mexico; N. Terunuma, H. Hayano, E. S. Kim, K. Kubo, T. Naito, J. Urakawa, S. Kashiwagi, and T. Okugi, in Proceedings of the International Conference on Accelerators and Large Experimental Physics Control Systems, Beijing, China, 1997 (KEK, Tsukuba, to be published), KEK Report No. 97-233.

[16] MINUIT Reference Manual, edited by F. James (CERN,
Geneva, 1994), Library Long Writeup D506.

[17] M. Sands, SLAC Report No. SLAC-AP-085, 1991.

[18] F. J. Decker, C. Adolphsen, W. J. Corbett, P. Emma, I. Hsu, H. Moshammer, J. T. Seeman, and W. L. Spence, in Proceedings of the 1991 IEEE Particle Accelerator Conference, San Francisco, 1991 (IEEE, New York, 1991), p. 905

[19] H. G. Hereward, Proceedings of the 1975 ISABELLE Summer Study, BNL, 1975 (BNL, Upton, NY, 1975), p. 555.

[20] E. Messerschmid and M. Month, Nucl. Instrum. Methods 136, 1 (1976).

[21] N. Terunuma, H. Hayano, E. S. Kim, K. Kubo, T. Naito, J. Urakawa, S. Kashiwagi, and T. Okugi, in Proceedings of the Sixth European Particle Accelerator Conference, Stockholm, 1998 (Institute of Physics, Bristol, UK, 1998), p. 481.

[22] H. Bruck and J. Le Duff, in Proceedings of the 5th International Conference on High Energy Accelerators, Frascati, Italy, 1965 (Comitato Nazionale per l'Energia Nucleare, Rome, 1966), p. 284.

[23] J. Le Duff, in Proceedings of the CERN Accelerator School, Fifth Advanced Accelerator Physics Course, Rhodes, Greece, 1993 (CERN, Geneva, 1995), p. 573.

[24] A. Piwinski, in Proceedings of the Ninth International Conference on High Energy Accelerators, Stanford, 1974 (SLAC, Stanford, CA, 1974), p. 405.

[25] T. Raubenheimer, SLAC Report No. SLAC-PUB-5790, 1992.

[26] SAD code is the accelerator design and simulation code developed by KEK accelerator theoretical group. See, for example, H. Hirata, in Proceedings of the Second Advanced ICFA Beam Dynamics Workshop, Lugano, Switzerland, 1988 (CERN, Geneva, 1988), p. 62. 